\title{
EFFECT OF SHORT TERM DIRECTIONAL SELECTION ON GENETIC VARIABILITY: EXPERIMENTS WITH DROSOPHILA MELANOGASTER
}

\author{
D. A. SORENSEN and W. G. HILL \\ Institute of Animal Genetics, West Mains Road, Edinburgh, EH9 3JN
}

Received 6.vii.81

\section{SUMMARY}

\begin{abstract}
Experimental checks on theoretical predictions of the build up of negative linkage disequilibrium with directional selection were made using abdominal bristle number in Drosophila melanogaster. Selection was practised for three generations before relaxation. Realized heritabilities and thus genotypic variances were estimated by divergent selection.

In one replicate, little change of variance occurred but in the other it increased substantially on relaxation. This result is compatible with a model of one or more genes of large effect at extreme frequencies in the base population. This is illustrated with Monte Carlo simulations. Interpretation of results was aided by considering the build up of negative disequilibrium.
\end{abstract}

\section{INTRODUCTION}

IN large populations, changes of genetic parameters caused by selection are due to changes in the frequencies of the genes affecting the trait and due to the generation of covariances between the frequencies of these genes at different loci, i.e., linkage disequilibrium.

The changes due to gene frequency changes are highly dependent on the distribution of gene effects and frequencies in the base population; little information is available on this in Drosophila and virtually none in other species. For a given amount of initial genetic variation, the larger the number of loci affecting the trait, the smaller the changes in genotypic variance due to gene frequency changes caused by selection. This type of argument together with a general impression obtained from the literature led people tentatively to suggest that parameters are not likely to change much during short term selection experiments. Consequently, the prediction of expected responses based on present heritability estimates may be valid for a period of five or more generations (Falconer, 1960).

Felsenstein (1965) and Bulmer $(1971,1974)$ have shown, however, that directional selection causes negative covariances between the frequencies of the genes at different loci, with a consequent reduction in genotypic variance, as predicted earlier on by Lush (1948). In contrast with the changes in variance due to gene frequency changes, the reduction in variance from linkage disequilibrium depends rather little on the number and effects of individual genes. This is so provided gene action is additive, gene effects are small and gene frequencies not extreme (Sorensen, 1980), and it is clearly independent of such factors when the number of loci is assumed to be infinite. Using this "infinitesimal model", Bulmer (1971) showed that the genotypic variance in the base population, $V_{G}=h^{2} \sigma^{2}$, is reduced after one cycle of directional selection, to $h^{2} \sigma^{2}\left[1-\frac{1}{2} i(i-x) h^{2}\right]$ in the offspring generation and a little more subsequently unless loci are 
tightly linked, where $h^{2}$ is the heritability, $\sigma^{2}$ is the phenotypic variance, $i$ the selection intensity and $x$ the corresponding truncation point of the standardized normal.

Previous directional selection experiments have not been designed specifically to observe the changes in variance due to disequilibrium and to check the theoretical models with animal experiments. The experiment described here was designed to do this, by selecting for bristle number in Drosophila for a few generations and then relaxing selection to allow for the breakdown of disequilibrium and see if heritability increased.

In checking the theory with organisms like Drosophila, the contribution to changes in genotypic variance from gene frequency changes can not be ignored. In order to provide a basis for interpreting the Drosophila data and to see the consequences of relaxing the assumption of an infinite number of loci on Bulmer's theory, we used Monte Carlo simulation with additive models involving a finite number of loci. The experimental results are reported first.

\section{Material AND Methods}

Lines were drawn from the Dahomey population of Drosophila melanogaster which had been kept in cages for eight years prior to the start of this experiment. Cultures were maintained at $25^{\circ} \mathrm{C}$ on standard Edinburgh medium. The character measured was the sum of the number of abdominal bristles on the fourth and fifth segments of males and fifth and sixth segment of females.

The experiment was run with two replicates and the design and line designation are shown in fig. 1. Flies were maintained in vials: in generation 0 , there were 30 full sib families, from each of which five males and five females were sampled and scored. Subsequently, there were 15 families, each providing 10 males and females for scoring. Out of the 150 scored of each sex, 15 of each sex were selected high or in both directions. The four generations without selection starting from $\mathrm{H} 3$ were maintained by sampling one male and female from each family and randomly mating in vials. In addition, flies were maintained during these four generations of

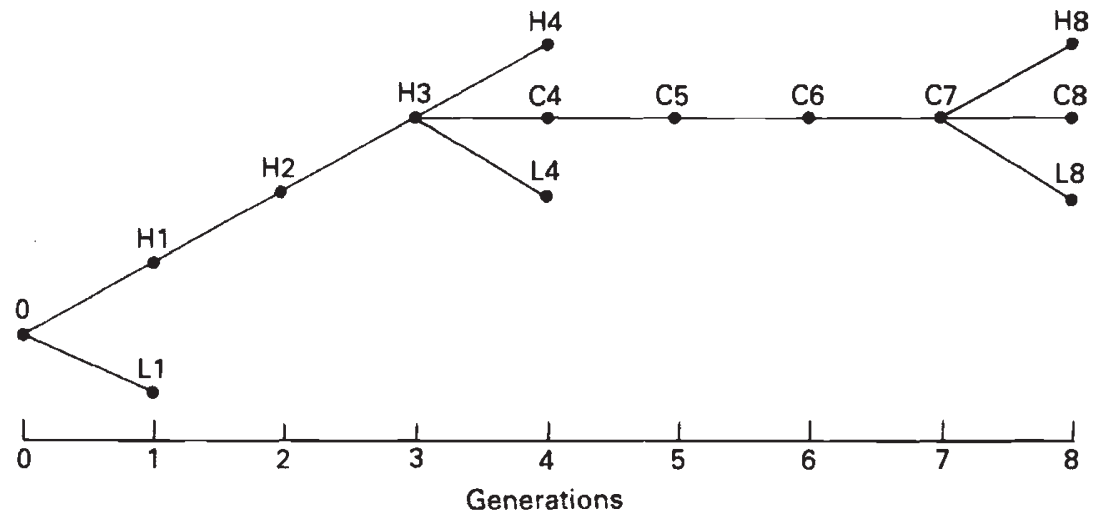

FIG. 1.-Design of experiment. The letters $\mathrm{H}, \mathrm{L}$ or $\mathrm{C}$ denote whether parents were selected for high bristle number, selected for low bristle number or randomly selected, respectively. 
relaxed selection, under crowded conditions in bottles to investigate any regression of the mean during random mating.

\section{EXPERIMENTAL RESULTS}

The means and other derived parameters are given in table 1 . Selection intensities are not tabulated; in terms of standard deviation they remained relatively unchanged during the experiment and were slightly smaller than the expected value of 1.75 obtained for normal tables; this is presumably due to the fact that spare flies were used to replace unsuccessful matings. Responses and realized heritabilities were computed from the divergence between high and low selected lines from the same source, divided by the sum of the selection differentials, and standard errors of realized heritabilities were computed from the formulae of Hill (1971). Genotypic variances were estimated as the product of the realized heritabilities and the corresponding phenotypic variance in the source population. A comparison between variance estimates at generation 3 and 7 gives the effect of breakdown of disequilibrium during relaxation; a comparison between 0 and 7 gives the effect of gene frequency changes.

TABLE 1

Mean of lines $(\overline{\mathrm{X}})$, estimates of genotypic variance $\left(\mathrm{V}_{\mathrm{G}}\right)$ and of single generation realized heritabilites $\left(h^{2}\right)$ for the Drosophila experiment. The figures in brackets are twice intra-class correlations between sibs obtained from the offspring of selected matings except for the estimate at $C 7$ which is obtained before selection operates

\begin{tabular}{|c|c|c|c|c|c|c|c|}
\hline \multirow[b]{2}{*}{$\begin{array}{c}\text { Source } \\
\text { line }\end{array}$} & \multirow[b]{2}{*}{$\begin{array}{c}\text { Derived } \\
\text { lines }\end{array}$} & \multicolumn{3}{|c|}{ Replicate I } & \multicolumn{3}{|c|}{ Replicate II } \\
\hline & & $\bar{X}$ & $V_{G}$ & $h^{2}$ & $\bar{X}$ & $V_{G}$ & $h^{2}$ \\
\hline & 0 & 35.99 & & & 36.22 & & \\
\hline 0 & $\begin{array}{l}\text { L1 } \\
\text { H1 }\end{array}$ & $\begin{array}{l}33 \cdot 70 \\
37 \cdot 88\end{array}$ & 3.63 & $0.42(0 \cdot 25)$ & $\begin{array}{l}33 \cdot 90 \\
37 \cdot 42\end{array}$ & $2 \cdot 96$ & $0.38(0.19)$ \\
\hline H3 & $\begin{array}{l}\mathrm{L} 4 \\
\mathrm{H} 4\end{array}$ & $\begin{array}{l}39 \cdot 81 \\
45 \cdot 28\end{array}$ & $5 \cdot 83$ & $0.45(0.33)$ & $\begin{array}{l}43 \cdot 10 \\
40 \cdot 50\end{array}$ & $2 \cdot 28$ & $0.26(0.20)$ \\
\hline $\mathrm{C} 7$ & $\begin{array}{l}\text { L8 } \\
\text { C8 } \\
\text { H8 }\end{array}$ & $\begin{array}{l}39 \cdot 65 \\
42 \cdot 32 \\
46 \cdot 92\end{array}$ & $7 \cdot 66$ & $0.59(0.56)$ & $\begin{array}{l}39 \cdot 85 \\
41 \cdot 55 \\
42 \cdot 26\end{array}$ & $2 \cdot 08$ & $0.26(0.11)$ \\
\hline Average $S E^{*}$ & & & & $0 \cdot 04(0 \cdot 10)$ & & & $0.04(0.12)$ \\
\hline Total response ${ }^{\dagger}$ & & $6 \cdot 46$ & & & & $4 \cdot 50$ & \\
\hline
\end{tabular}

The two replicates differ very markedly. In replicate I, the heritability increased very slightly during the three generations of selection for high bristle number and there was a substantial rise during random mating. In replicate II, heritability tended to decline during selection and did not increase after the period of random mating.

The data allowed estimation of intra-class correlations between full sibs. These were obtained separately from the offspring generation of the 
high and low matings. Both estimates were pooled and the results are shown in brackets in table 1 . The standard errors are large but the estimates are consistently smaller than the contemporary realized heritabilities, except for the estimate of replicate I obtained at the end of the period of random mating. These results are to be expected from theory, as intra-class correlations obtained from populations undergoing selection, yield estimates of heritability which are biased downwards, the bias being due to linkage disequilibrium (Robertson, 1977). During the period of relaxed selection, there was no apparent change in mean either for the populations maintained in vials (table 1) or in crowded conditions in bottles (not shown).

\section{MONTE CARLO SIMUlation RESUlts}

Two additive genetic models were run, each involving about 30 loci with two alleles each, equally spaced on three chromosomes with recombination fraction between adjacent loci of $0 \cdot 1$. The genotypic value of an individual is the sum of the values at each locus. In model I, there were three loci of large effect on the trait ( $1 \cdot 10$ phenotypic standard deviations), one at very low initial frequency $(0.01)$, and the other two at high frequency $(0.99)$. The remaining 27 loci were divided into three groups of 8,11 , and 8 loci, respectively, whose initial frequencies were $0 \cdot 40,0 \cdot 50$, and $0 \cdot 60$, and standardized gene effects were about $0 \cdot 16 \sigma$. Model II was similar to model I, except that the locus of large effect at low frequency was absent. In both models, 15 males and females were selected out of 150 scored in each sex and mated at random. Gene frequencies were calculated for each locus each generation, and from these, summing over loci, a value of the genic variance was obtained assuming Hardy-Weinberg and linkage equilibrium. The genotypic variance was obtained from the distribution of genotypic values. The difference between the genotypic variance and the genic variance estimates the effect due to disequilibrium (ignoring small departures from Hardy-Weinberg equilibrium). Single generation realized heritabilities were obtained by dividing the response by the selection differential. Selection was carried out for three generations, followed by four cycles of relaxation as in the Drosophila experiment. Each model was replicated 16 times.

Results are shown in table 2, together with predictions based on the assumption of an infinite number of loci. In model $I$, as the rare gene of large effect increases in frequency, the genic variance rises sharply, but this effect on the genotypic variance is partly masked by the generation of a large amount of negative disequilibrium. Upon relaxation, most of the disequilibrium breaks down and the genotypic variance and heritability increase. In model II, due to the absence of the gene of large effect, the genic and the genotypic variance decrease during selection and the amount of disequilibrium produced is much smaller than in model $\mathrm{I}$, such that after relaxation, no increase in heritability is detected. There is thus a considerable difference in the overall change in the genotypic variance and the amount of disequilibrium generated in both models. However, when allowance is made for the difference in gene frequencies and effects, the results are very similar in models I and II and in the infinitesimal model. This is illustrated by the ratio of genotypic to genic variance, which remains at a value of about 80 per cent after the first cycle of selection. 
$: \frac{2}{2} \cdot \frac{2}{2}$

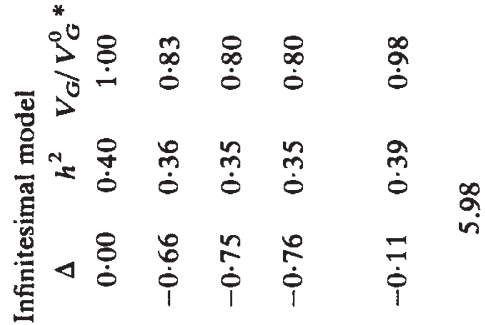

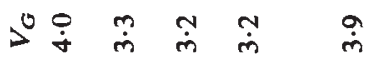

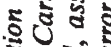

เป

อ

के

政事

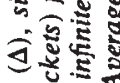

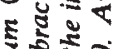

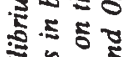

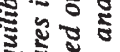

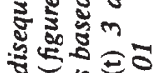
ชิ 5 :

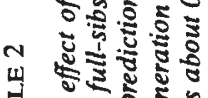

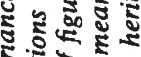

จ

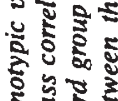

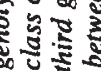

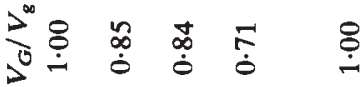

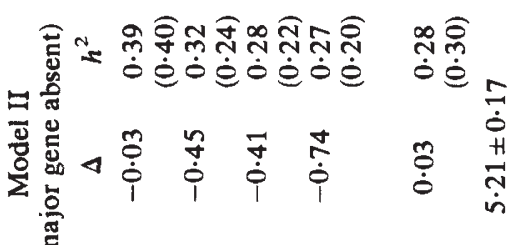

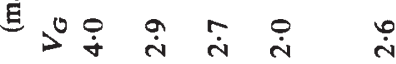

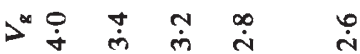

का है

论

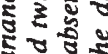

จ

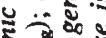

戠文

पर ह ह

ริธ

จ. จั จ

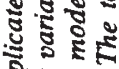

ข้ํํำ

응

吨

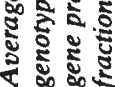

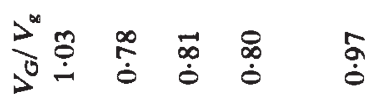

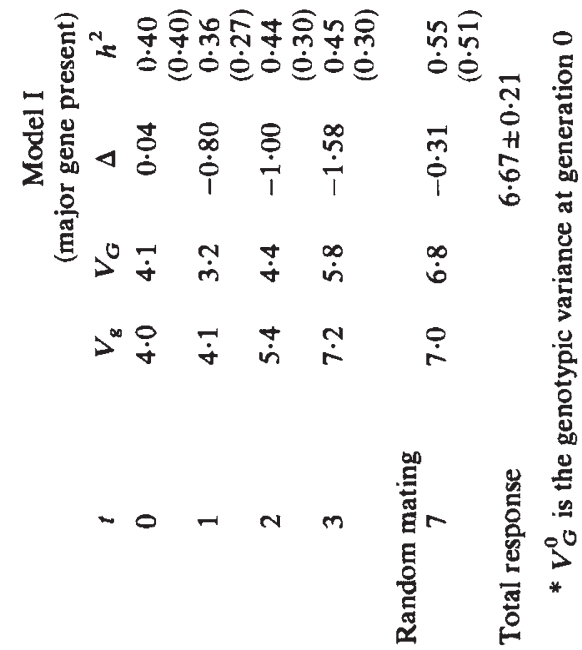


Twice the intra-class correlation between full sibs obtained in a manner analogous to the Drosophila experiment are shown in brackets. In common with the Drosophila data, the estimates are biased downwards during the period of selection.

\section{Discussion}

The experiment reported here was designed to observe whether the genotypic variance declined during selection due to the generation of disequilibrium as anticipated by Bulmer (1971). Under the infinitesimal model, the predicted decline in heritability is of about 12 per cent (see table 2). Based on formulae by Hill (1971), the standard error of the realized heritability estimates is about 10 per cent per replicate or about 7 per cent for a pair of replicates, and therefore, the size of our experiment is not large enough to detect such a change.

The simulation results (table 2 ) show, however, that with a finite number of loci, the generation of disequilibrium contributes very significantly to changes in the genotypic variance, but the magnitude of this contribution depends on initial gene effects and frequencies. If genes of large effect at low frequencies are present in the initial sample, the genic variance will rise sharply as selection operates, but this effect on the genotypic variance will only be detected after a significant proportion of the large negative disequilibrium generated breaks down after random mating.

Models I and II of the simulation can be envisaged as two replicates drawn from a population in which the trait of interest is determined by a small number of loci of comparatively large effect with "plus" alleles initially at extreme frequencies. The loci of smaller effect are more numerous and have intermediate frequencies. In model $\mathrm{I}$, the rare major gene is picked up and it is absent in model II. A characteristic feature of this model is that it leads to pronounced variation between replicated selected lines of moderate size, since a fair number of samples would include or fail to include the major genes (James, 1971). This type of model has been postulated on several occasions to explain the behaviour of selected lines of Drosophila bristle numbers (Clayton et al., 1957; Sen and Robertson, 1964; Frankham et al., 1968; Yoo, 1980).

Without pressing the comparison too far, we want to suggest this model as a plausible explanation for the results obtained from the Drosophila experiment, our conjecture being that in replicate $\mathrm{I}$, as in model $\mathrm{I}$, the major gene is present but it is absent in replicate II. Thus, the overall response, the change in realized heritabilities, and the intra-class correlations in replicates I and II follow similar patterns to the value of these parameters in models I and II, respectively.

It is difficult to say whether the difference between the heritability estimates of generations 3 and 7 in replicate $I$ is significant since the standard errors will tend to be underestimated under this model using Hill's formula, The average standard deviation of the realized heritabilities in model I obtained from the simulation is of about 0.065 , which casts some doubt about the statistical significance of this difference, even if allowance is made for the fact that the estimates are correlated. Therefore all we can say is that this simple model is compatible with the data.

Our results suggest that, at least for Drosophila bristles, the predictions of changes in variance using the infinitesimal model are not very useful 
because gene effects may not be sufficiently small for gene frequency changes to be negligible. Nevertheless, we have shown that the generation of linkage disequilibrium can play a major role in the changes of variance during selection.

\section{REFERENCES}

BULMER, M. G. 1971. The effect of selection on genetic variability. Amer. Nat., 105, 201-211. BULMER, M. G. 1974. Linkage disequilibrium and genetic variability. Genet. Res. 23, 281-289.

CLAYTON, G. A., KNIGHT, G. R., MORRIS, J. A., AND ROBERTSON, A. 1957. An experimental check on quantitative genetical theory. III. Correlated responses. J. Genet., 55, 171-180.

FALCONER, D. S. 1960. Introduction to Quantitative Genetics. Oliver and Boyd. Edinburgh. FELSENSTEIN, J. 1965. The effect of linkage on directional selection. Genetics, 42, 349-363. FRANKHAM, R., JONES, L. P., AND BARKER, J. S. F. 1968. The effects of population size and selection intensity in selection for a quantitative character in Drosophila. III. Analysis of the lines. Genet. Res. 12, 267-283.

HILL, W. G. 1971. Design and efficiency of selection experiments for estimating genetic parameters, Biometrics, 27, 293-311.

JAMES, J. W. 1971. The founder effect and response to artificial selection. Genet. Res. 16, $241-250$.

LUSH, J. L. 1948. The Genetics of Populations. Mimeograph.

ROBERTSON, A. 1977. The effect of selection on the estimation of genetic parameters. Z. Tierz. Zuchtbiol., 94, 131-135.

SEN, B. K., AND ROBERTSON, A. 1964. An experimental examination of methods for the simultaneous selection of two characters using Drosophila melanogaster. Genetics, 50 , 199-209.

SORENSEN, D. A. 1980. The effect of truncation selection on genetic variability. Ph.D. Thesis, Univ. of Edinburgh.

YOO, B. H. 1980. Long term selection for a quantitative character in large replicate populations of Drosophila. I. Response to selection. Genet. Res, 35, 1-17. 